\title{
Myelin and Axonal Damage in Normal-Appearing White Matter in Patients with Moyamoya Disease
}

\author{
(D). Hara, (D) M. Hori, (D) A. Hagiwara, (D). Tsurushima, (D). Tanaka, (D). Maehara, (D). Aoki, and (D). Nariai
}

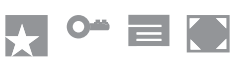

\begin{abstract}
BACKGROUND AND PURPOSE: Although chronic ischemia is known to induce myelin and axonal damage in animal models, knowledge regarding patients with Moyamoya disease is limited. We aimed to investigate the presence of myelin and axonal damage in Moyamoya disease and their relationship with cognitive performance.

MATERIALS AND METHODS: Eighteen patients with Moyamoya disease (16-55years of age) and 18 age- and sex-matched healthy controls were evaluated with myelin-sensitive MR imaging based on magnetization transfer saturation imaging and 2-shell diffusion MR imaging. The myelin volume fraction, which reflects the amount of myelin sheath; the g-ratio, which represents the ratio of the inner (axon) to the outer (axon plus myelin) diameter of the fiber; and the axon volume fraction, which reflects axonal components, were calculated and compared between the patients and controls. In the patients with Moyamoya disease, the relationship between these parameters and cognitive task-measuring performance speed was also evaluated.
\end{abstract}

RESULTS: Compared with the healthy controls, the patients with Moyamoya disease showed a significant decrease in the myelin and axon volume fractions $(P<.05)$ in many WM regions, while the increases in the g-ratio values were not statistically significant. Correlations with cognitive performance were most frequently observed with the axon volume fraction $(r=0.52-0.54$; $P<.03$ in the right middle and posterior cerebral artery areas) and were the strongest with the g-ratio values in the right posterior cerebral artery region $(r=0.64 ; P=.004)$.

CONCLUSIONS: Myelin-sensitive MR imaging and diffusion MR imaging revealed that myelin and axonal damage exist in patients with Moyamoya disease. The relationship with cognitive performance might be stronger with axonal damage than with myelin damage.

ABBREVIATIONS: $\mathrm{ACA}=$ anterior cerebral artery; $\mathrm{AVF}=$ axon volume fraction; GRAPPA = generalized autocalibrating partially parallel acquisition; MMD = Moyamoya disease; $\mathrm{MVF}=$ myelin volume fraction; $M T$ sat $=$ magnetization transfer saturation; $\mathrm{PCA}=$ posterior cerebral artery; $V_{i c}=$ intracellular volume fraction; $V_{\text {iso }}=$ isotropic volume fraction; $T M T=$ Trail-Making Test; WAIS $=$ Wechsler Adult Intelligence Scale

C hronic ischemia induced in animal models is known to damage the structure of myelin, which wraps around axons to provide the efficient conduction of neural signals, and axons. ${ }^{1}$ Few previous studies have directly investigated whether chronic

Received March 29, 2020; accepted after revision June 5.

From the Department of Neurosurgery (S.H., Y. Tanaka, T.M., T.N.), Tokyo Medical and Dental University, Tokyo, Japan; Department of Radiology (S.H., M.H., A.H., Y.T., S.A.), Juntendo University, Tokyo, Japan; Department of Diagnostic Radiology (M.H.), Toho University Omori Medical Center, Tokyo, Japan; and Department of Radiology (Y. Tsurushima), Kenshinkai Tokyo Medical Clinic, Tokyo, Japan.

This work was supported by Grants-in-Aid for Scientific Research "KAKENHI," the Japan Society for the Promotion of Science (grants 19K18406 and 16H06280), and a research grant (2017-2020) from the Japanese Society of Neuroradiology.

Preliminary results previously presented at: Annual Meeting of The Japanese Society of Neuroradiology, February 16-18, 2018; Tsukuba, Japan; and Joint Annual Meeting of the International Society for Magnetic Resonance in Medicine and the European Society for Magnetic Resonance in Medicine and Biology, June 16-18, 2018; Paris, France. ischemia in humans also damages the myelin structure, such as in patients with Moyamoya disease (MMD).

MMD is a progressive cerebrovascular disease that mainly affects children and young adult patients in contrast to atherosclerotic cerebrovascular diseases, ${ }^{2}$ which cause chronic hemodynamic impairment characterized by strong perfusion delay. ${ }^{3,4}$ Previous diffusion MR imaging studies have suggested that in vivo microstructural damage might exist in this disease population and might be related to cognitive function. ${ }^{5-7}$ However,

Please address correspondence to Shoko Hara, MD, Department of Neurosurgery, Tokyo Medical and Dental University, 1-5-45 Yushima, Bunkyo-ku, Tokyo 113-8519, Japan; e-mail: shara.nsrg@tmd.ac.jp

\footnotetext{
- Indicates open access to non-subscribers at www.ajnr.org

$\equiv$ Indicates article with supplemental on-line appendix and tables.

Indicates article with supplemental on-line photos.

http://dx.doi.org/10.3174/ajnr.A6708
} 
because myelin contains few free water molecules and the water protons of macromolecules in the myelin sheath and water protons between the phospholipid bilayers have a short transverse relaxation time ( $\mathrm{T} 2 \approx 10 \mathrm{~ms}$ ), the signal is theoretically negligible in typical diffusion MR imaging acquisitions using long TEs ( $\approx 50-100 \mathrm{~ms}){ }^{8}$ Hence, diffusion MR imaging cannot directly assess myelin damage, and the results of these studies provide indirect evidence of myelin damage in MMD. To the best of our knowledge, no previous studies, including postmortem case reports, have directly investigated whether myelin damage exists in patients with MMD.

We hypothesized that myelin damage exists in patients with MMD and is related to cognitive function. To investigate this hypothesis, we applied an MR imaging technique called magnetization transfer saturation (MTsat), ${ }^{9}$ which sensitizes water molecules bound to macromolecules and enables the quantitative estimation of myelin in a clinically feasible scanning time. This technique has been shown to be clinically useful in patients with other neurologic disorders. ${ }^{10,11}$ The relationship between the axon size and myelin thickness can be captured by a parameter called the myelin g-ratio, which is defined as the ratio of the inner (axon) to the outer (axon plus myelin) diameter of the nerve fiber (On-line Fig 1; see the illustration in the box). ${ }^{8,12}$ Thus, we combined MTsat with diffusion MR imaging, which can evaluate the presence of axonal damage, ${ }^{13}$ to calculate the g-ratio. Because the destruction of myelin could lead to a decrease in the nerve-conduction velocity, ${ }^{14}$ we hypothesized that decreases in myelin and increases in the g-ratio correlated with reductions in processing speed.

\section{MATERIALS AND METHODS}

\section{Study Protocol and Background of the Participants}

This prospective observational study was approved by the ethics committees of Tokyo Medical and Dental University (M20002302) and Juntendo University (16-100), with registration to the University Hospital Medical Information Network Clinical Trials Registry (UMIN-CTR ID: 000027949). The inclusion criteria were patients diagnosed with MMD according to the diagnostic guidelines, ${ }^{15}$ 16-64 years of age, who were able to undergo MR imaging without sedative agents and had no neurologic deficits affecting the scores of neurophysiological tests, with a modified Rankin Scale score of $0-2$. The exclusion criteria were cortical or large ( $>10 \mathrm{~mm}$ ) WM infarcts visible on routine MR imaging sequences.

Between May 2017 and December 2019, eighteen patients (4 males; 17-55 years of age; average, 36 years; all right-handed), including 9 postoperative patients, participated in this study (details are provided in On-line Table 1 and the On-line Appendix). All patients underwent a series of MR imaging scans and a cognitive battery within an interval of $0-20$ days ( 7 days on average; On-line Table 2).

During the same period, 18 age- and sex-matched healthy subjects with no history of neurologic or systematic diseases (4 males; 16-44 years of age; average, 35 years) were recruited and evaluated by the same MR imaging protocol.

\section{Cognitive Assessments}

The cognitive battery (the details are provided in the On-line Appendix) included the Processing Speed Index and Trail-
Making Test parts A and B (TMT-A and -B). The Processing Speed Index is an index measuring visual motor speed in the Wechsler Adult Intelligence Scale (WAIS-III). TMT-A and -B assess the speed of information processing and executive functioning, respectively. The results of TMT-A and -B were converted to $z$ scores using the reported normal ranges of each age group.

\section{MR Imaging Acquisition}

All MR imaging data were acquired using a 3T system (Magnetom Skyra; Siemens) equipped with a 32-multichannel receiver head coil. The total acquisition time of the entire protocol, including T1WI, MRA, FLAIR, and SWI (acquisition parameters provided in the On-line Appendix), was approximately 30 minutes.

Three sagittal 3D multiecho fast low-angle shot sequences were performed with predominant magnetization transfer-, proton density-, and T1-weighting $(\mathrm{TR}=24 / 24 / 10 \mathrm{~ms}, \mathrm{TE}=1.85 /$ $1.85 / 1.85 \mathrm{~ms}$, flip angle $=5^{\circ} / 5^{\circ} / 13^{\circ}$, matrix $=128 \times 128$, section number $=128$, parallel acquisition techniques $=$ generalized autocalibrating partially parallel acquisition [GRAPPA] acceleration factor $=2, \mathrm{FOV}=200 \mathrm{~mm}$, voxel size $=1.56 \times 1.56 \times$ $1.56 \mathrm{~mm}$ ). For the magnetization transfer-weighted images, excitation was preceded by an off-resonance Gaussian-shaped radiofrequency pulse (frequency offset from water resonance $=$ $1200 \mathrm{~Hz}$, bandwidth $=192 \mathrm{~Hz}$, pulse duration $=9.984 \mathrm{~ms}$, and nominal flip angle $=500^{\circ}$ ). The acquisition times were 2 minutes 23 seconds, 2 minutes 23 seconds, and 1 minute. Two additional $\mathrm{B}_{1}$ maps using coronal echo-planar imaging with nominal $10^{\circ}$ and $20^{\circ}$ flip angles were acquired within a short acquisition time (approximately 10 seconds each).

The diffusion-weighted images were acquired using a fat-saturated single-shot echo-planar imaging sequence along 30 and 60 motion-probing gradient directions with $b$ values of 700 and $2850 \mathrm{~s} / \mathrm{mm}^{2}, \mathrm{TR}=4500 \mathrm{~ms}, \mathrm{TE}=105 \mathrm{~ms}$, flip angle $=90^{\circ}$, matrix $=100 \times 90$, number of slices $=90$, voxel size $=2 \times 2 \times 2$ $\mathrm{mm}$, multiband factor $=2$. Several volumes of non-diffusionweighted images were also acquired with reversed phase-encoded blips, resulting in pairs of images with distortions along opposite directions. The acquisition times were approximately 8 minutes 38 seconds for the main images and 1 minute 37 seconds for the reversed phase-encoded blips.

\section{Postprocessing of the MR Imaging Data}

The postprocessing of the MR imaging data (On-line Fig 1) was conducted using Matlab 2014a (MathWorks). The data were exported from the scanner in DICOM format and converted to NIfTI format using dicm2nii (https://www.github.com/ xiangruili/dicm2nii). Images with magnetization transfer-, proton density-, and T1-weighting were converted to the MTsat index using a previously described method, ${ }^{9}$ while correcting for small higher order dependencies of the MTsat on the local radiofrequency transmit field using $B_{1}$ maps. ${ }^{16}$ The MTsat index was multiplied by 0.1 ; this calibration calculated the myelin volume fraction (MVF), revealing that the amount of myelin sheath in the splenium of the corpus callosum in the healthy volunteers was $0.3-0.4$ and that the g-ratio was $0.7-0.8$, consistent with the 
values reported in previous studies. ${ }^{8,17,18}$ Values under 0 and above 1 were considered errors and changed to 0 .

Based on the paired non-diffusion-weighted images (ie, $b=0$ $\mathrm{s} / \mathrm{mm}^{2}$ ), the susceptibility-induced off-resonance field was estimated using a method similar to previously described procedures $^{19,20}$ implemented in the FMRIB Software Library (FSL; http://www.fmrib.ox.ac.uk/fsl) $)^{21}$ to correct for susceptibilityinduced distortions in the diffusion-weighted images. Then, the diffusion data were fitted to the neurite orientation dispersion and density imaging model using the NODDI Matlab toolbox, Version $0.9^{13}$ (https://www.nitrc.org/projects/noddi_toolbox/) and converted to the intracellular volume fraction $\left(V_{i c}\right)$ and isotropic volume fraction $\left(V_{i s o}\right)$. The axon volume fraction (AVF), which reflects the axonal components, and the g-ratio were calculated from MVF, $V_{i c}$, and $V_{i s o}$ using the following equations: ${ }^{8,22}$

$$
\begin{aligned}
\mathrm{AVF}= & (1-\mathrm{MVF}) \times\left(1-V_{\text {iso }}\right) \times V_{i c}, \\
& \mathrm{~g}-\text { ratio }=\sqrt{\frac{\mathrm{AVF}}{\mathrm{MVF}+\mathrm{AVF}}} .
\end{aligned}
$$

WM segmentation was performed on 3D-T1WI using the standard segmentation tool in SPM12 (http://www.fil.ion.ucl.ac.uk/ spm/software/spm12/). WM masks with a probability threshold of 0.9 were created and applied to the MVF, AVF, and g-ratio maps to minimize partial volume effects. WM lesions visible on FLAIR and $b=0 \mathrm{~s} / \mathrm{mm}^{2}$ images were manually deleted from each map.

\section{Calculation of Regional Values}

The WM labels of the Harvard-Oxford cortical and subcortical probabilistic structural atlases (http://ftp.nmr.mgh.harvard.edu/ pub/dist/freesurfer/tutorial_packages/centos6/fsl_507/doc/wiki/ Atlases.html) ${ }^{23}$ (threshold of 25\%) distributed with FSL were divided into 6 vascular regions (anterior, middle, and posterior cerebral arteries [ACA, MCA, and PCA] of the right and left sides) using the vascular territorial atlas (https://figshare.com/articles/ ATT_based_flow_territories/1488674) as a reference. ${ }^{24}$ These labels were converted to each subject's space by linear (FLIRT; http://www.fmrib.ox.ac.uk/fsl/fslwiki/FLIRT) and nonlinear (FNIRT; http://fsl.fmrib.ox.ac.uk/fsl/fslwiki/FNIRT) Image Registration Tools implemented in $\mathrm{FSL}^{21}$ and were used as ROIs to extract the MVF, AVF, and g-ratio values of each region (On-line Fig 1).

\section{Statistical Analysis}

Unpaired $t$-tests were used to compare the regional values of the MVF, AVF, and g-ratio between the patients with MMD and the healthy controls. Pearson correlation coefficients were calculated between regional metrics and cognitive scores in patients with MMD. The analyses were performed using JMP, Version 12.0.1 (SAS Institute), and $P<.05$ (with and without Bonferroni correction for comparison of 3 parameters) was regarded as statistically significant.

\section{RESULTS}

Representative cases are shown in Fig 1 and On-line Fig 2. As shown in Fig 1, a decreased MVF and decreased AVF were observed in the affected hemisphere, while the increase in the gratio was less prominent. As shown in On-line Fig 2, a decreased MVF was observed in the postoperative hemisphere as well, which had an improved hemodynamic condition at the time of the study. The decrease in AVF was unclear, and the g-ratio seemed to be slightly increased in the postoperative hemisphere.

\section{Comparison of the Regional Values between Patients and Controls}

The regional MVF values in patients with MMD were significantly lower than those in healthy controls in all regions (Fig 2). The regional AVF values in bilateral MCA regions in the patients were significantly lower than those in the healthy controls. The g-ratio tended to be higher in patients than the controls in all regions, but the difference was not statistically significant.

\section{Correlation between the Regional Values and Cognitive Assessments}

Across the 6 regions evaluated, the right PCA area showed the highest number of significant correlations between the MR imaging metrics and cognitive performance. The AVF in the right PCA area was significantly correlated with the Processing Speed Index and TMT-A and -B (Fig 3). The g-ratio in the right PCA area and the left ACA area was positively correlated with the Processing Speed Index (Fig 2 and On-line Table 3; $r=0.49$, $P=.04$ in left ACA area). In the right MCA area, the MVF and AVF were negatively correlated with the TMT-B scores (On-line Table 2; $r=-0.49, P=.04$ and $r=-0.55, P=.02$, respectively). No significant correlations were observed between other combinations of the MR imaging metrics and cognitive performance (On-line Table 2).

The details of the methods and results are provided in the On-line Appendix, and the full dataset is available from the corresponding author on reasonable request.

\section{DISCUSSION}

Consistent with our hypothesis, we found statistically significant decreases in the MVF and AVF in the patients with MMD compared with healthy controls. Meanwhile, the g-ratio in the patients tended to be higher than that in the controls but was not statistically significant in any region. The significant decrease in the AVF and MVF suggests that concomitant axonal and myelin damage might have buffered the difference in the g-ratio between the patients and controls. This finding is consistent with a previous animal study suggesting that myelin damage precedes axonal damage, ${ }^{25}$ and a diffusion MR imaging study showing that myelin damage is more widespread than axonal damage in patients with MMD without an operation. ${ }^{7}$ The decrease in the MVF in our patients seems to be more widespread and prominent than the decrease in the AVF; therefore, it is possible that myelin breakdown precedes axonal destruction under chronic ischemic conditions in patients with MMD.

Unexpectedly, we found significant correlations between the MVF or g-ratio values and cognitive performance in only a few areas. A previous study showed that myelin damage in patients with stroke is correlated with motor impairment and function; ${ }^{26}$ 


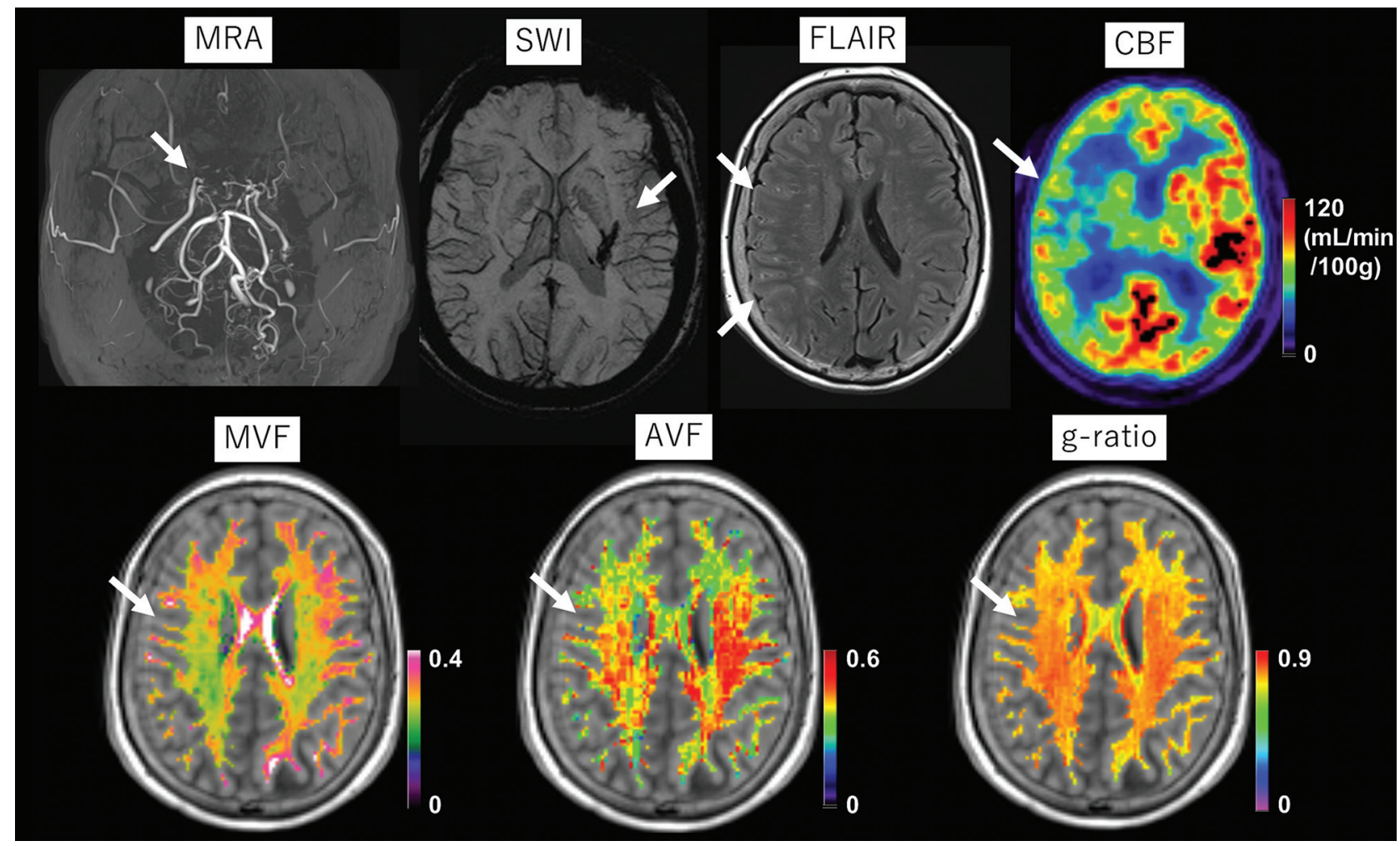

FIG 1. A 55-year-old female patient with MMD who had a history of thalamic hemorrhage $>20$ years ago (arrow on SWI). During the annual follow-up, though she remained asymptomatic, the arterial stenosis on the right side gradually progressed (arrow in MRA), and an ivy sign emerged on the right hemisphere (arrows on FLAIR). $\left[{ }^{15} \mathrm{O}\right]$-gas PET reveals decreased CBF on the right side (arrow). The myelin volume fraction and axon volume fraction are visually decreased in the right hemisphere (arrows). The right-left difference in the g-ratio (arrow) is not as evident as the differences in the MVF and AVF.
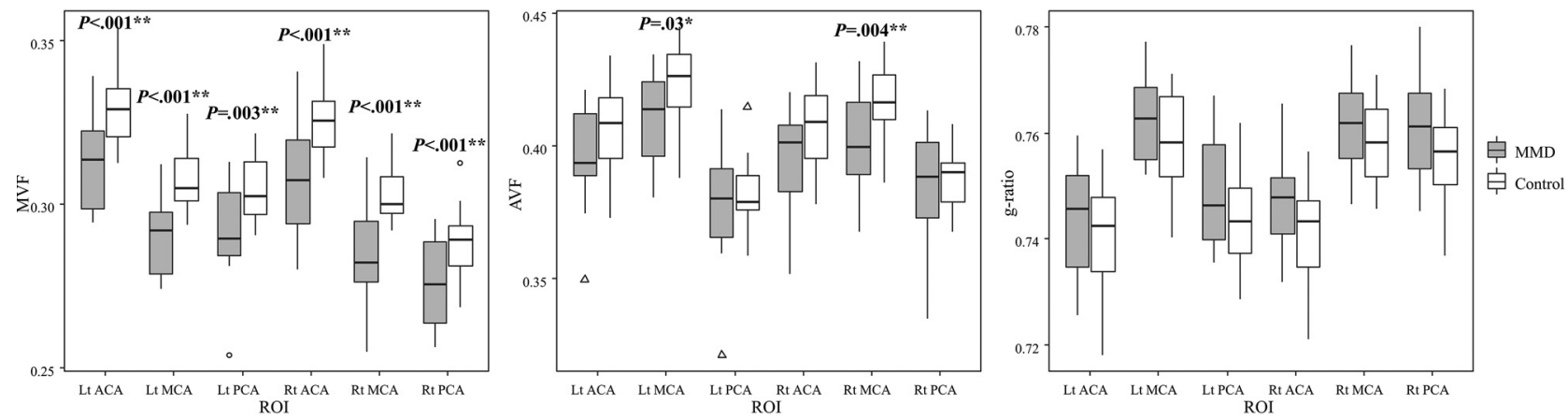

FIG 2. Comparison of the myelin volume fraction, axon volume fraction, and g-ratio between the patients and healthy controls. The asterisk indicates $P<.05$; double asterisks, $P<.05$ (Bonferroni correction for comparing 3 parameters). Lt indicates left; Rt, right.

therefore, we presumed that myelin damage (decreased MVF and increased g-ratio) in patients with MMD is related to cognitive tasks measuring processing speed that must reflect nerve-conduction velocity. ${ }^{14}$ The difference between patients with stroke without MMD and patients with MMD might be partially explained by the fact that ischemic damage in patients with stroke without MMD is sudden and focal, while ischemic damage in patients with MMD is chronic and diffuse. ${ }^{6,7}$ Perhaps because of the uniform and widespread decrease in the myelin content, the MVF values showed relatively small variation in patients compared with the AVF values and, thus, did not correlate with various degrees of cognitive performance.
In addition to the few correlations among the MVF, g-ratio, and cognitive performances, the observed correlation between the Processing Speed Index and the g-ratio was positive, a finding inconsistent with our hypothesis. As discussed above, the decrease in the MVF and the increase in the g-ratio might precede the decrease in the AVF and the decrease in the g-ratio. ${ }^{7,25}$ Moreover, in patients with MMD, both the cortex and white matter are affected by chronic ischemia, ${ }^{5-7,27,28}$ and while direct ischemic damage to white matter might result in more myelin damage than axonal damage, cortical damage must cause both myelin and axonal damage due to the decrease in axonal fibers radiating from the affected cortex. Thus, the presence of axonal 

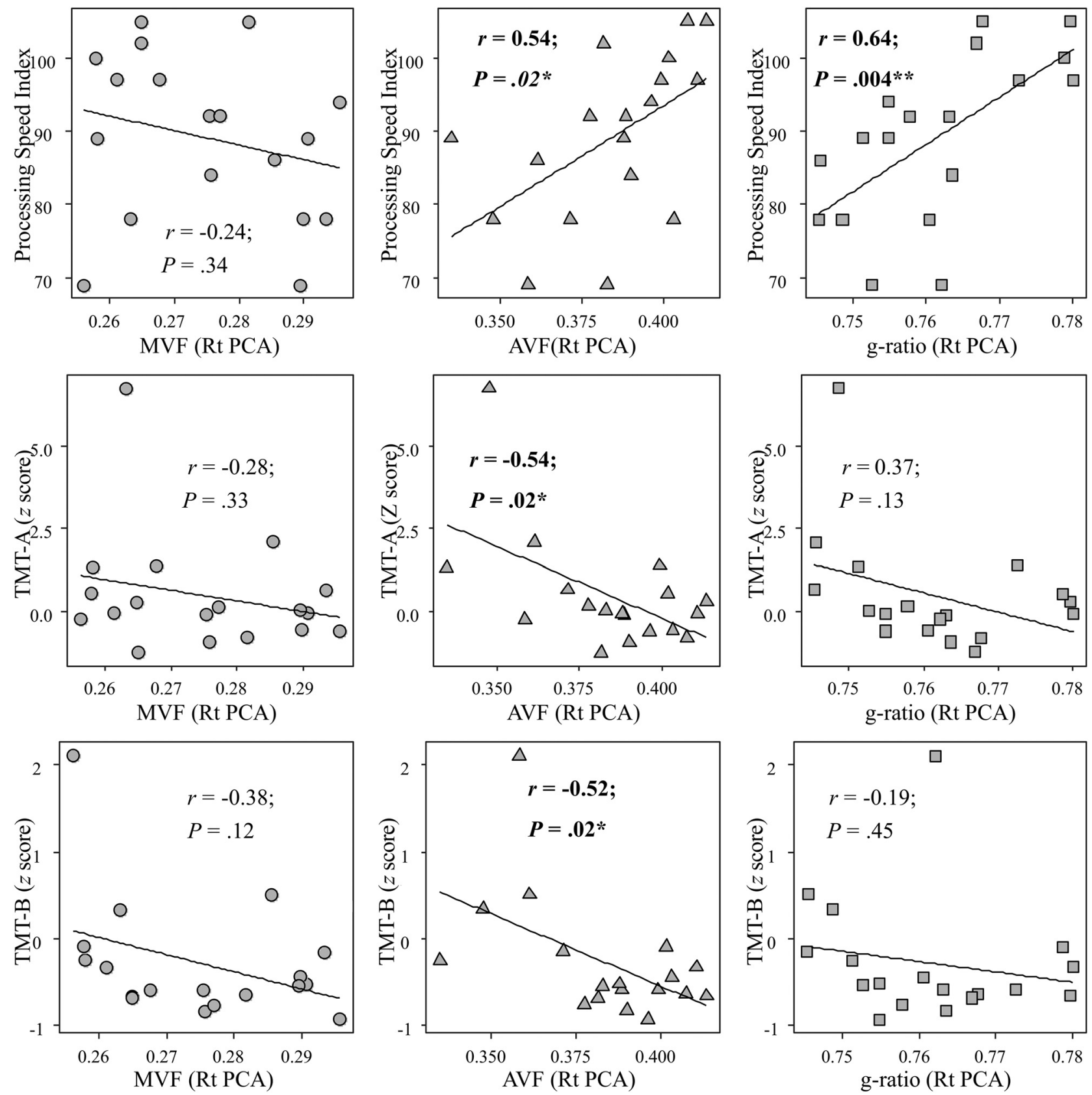

FIG 3. Correlations between cognitive performance and the myelin volume fraction, axon volume fraction, and g-ratio in the right PCA area of patients with Moyamoya disease. The asterisk indicates $P<.05$; double asterisks, $P<.05$ (Bonferroni correction for comparing 3 parameters).

damage might reflect more global damage than myelin damage in this disease population. This assumption is consistent with the finding that the decrease in the AVF is not as widespread as the decrease in the MVF in our patients. The lack of a correlation with the MVF and the positive correlation with the AVF might lead to the positive correlation between the g-ratio and the Processing Speed Index. The decrease in the AVF causing the increase in the g-ratio might be more critical for cognitive function in patients than the decrease in the MVF causing the increase in the g-ratio, thus resulting in a stronger correlation with the gratio and the Processing Speed Index than the AVF.

The strongest correlation between the MR imaging metrics and cognitive performance in the patients with MMD was found in the right PCA area. This finding was unexpected because generally, ischemic damage to the frontal lobe is regarded as the cause of cognitive decline in patients with MMD, and all our patients were right-handed and the dominant hemisphere is supposedly the left side. Regarding laterality, some studies reported that the right side of the brain is related to the cognitive performance of patients with $\mathrm{MMD},{ }^{29,30}$ though the reason for laterality is unclear. The laterality of the result might have been affected by our finding that PCA lesions more frequently occurred on the right side than on the left side, though the difference was not statistically significant (On-line Table 1: $9 / 18$ versus $6 / 18, P=.28$ by a paired $t$ test). Regarding the posterior part of the brain and cognition, some studies have reported a relationship between the 
posterior part of the brain and cognitive function in patients with MMD. ${ }^{6,30}$ We speculated that the significant correlation only in the PCA area is due to the variation in the ischemic condition and the parametric values in this area, while the ACA and MCA areas were uniformly affected in many patients and had a relatively smaller variation in the parametric values. The nonsignificant correlation among the cognitive tasks measuring working memory, which is regarded as a frontal lobe function, is also consistent with this assumption ${ }^{27}$ (Online Tables 2 and 3).

Although approximately half of the patients in this study were in a postoperative status and clinically stable without symptoms at the time of the study (eg, the patient in Fig 3), the differences in the MVF and AVF between the patients and healthy controls were statistically significant in many regions. These results suggest that ischemic damage to myelin and axons in patients with MMD might be, at least partially, irreversible. ${ }^{6,27}$ Postoperative improvements in microstructural integrity evaluated by diffusion MR imaging are reported in patients with $\mathrm{MMD}^{28}$ and carotid artery stenosis, ${ }^{31}$ but whether these observed changes are due to an increase in axon integrity, such as remyelination, or a decrease in interstitial fluid ${ }^{32}$ remains unclear. Compared with diffusion MR imaging, which indirectly assesses the presence of myelin damage by evaluating increased water diffusion in the brain parenchyma, the MTsat method has the strength of being directly sensitive to myelin. To clarify whether microstructural damage to myelin and axons is reversible, we are currently conducting a prospective study to re-evaluate preoperative patients 1 year after bypass surgery using the same MR imaging protocol. If microstructural damage is irreversible, surgical revascularization to restore hemodynamic impairments might be required to protect patients from future cognitive dysfunction, even among those without any symptoms.

Limitations of this study include the small sample size and heterogeneous patient backgrounds, and we did not evaluate the cognitive performance of the healthy controls; thus, the results, especially those related to cognitive function, should be considered preliminary. Most of our patients were previously surgically treated; thus, the relationships between the cerebral hemodynamics and microstructural parameters were difficult to assess because many patients showed improved hemodynamic conditions, such as the patient in On-line Fig 2. However, our study revealed that postoperative patients who have improved hemodynamic conditions might still have myelin damage. Because many myelin-specific imaging techniques are available, it is possible that different types of myelin-sensitive imaging might show different results. ${ }^{33-36}$ Given the complicated postprocessing, myelin imaging may not be practical for routine application in clinical settings compared with conventional diffusion parameters (On-line Table 2). Despite these limitations, this study is the first to directly investigate myelin damage using a myelin-sensitive MR imaging technique in adult patients with MMD and its relationship with cognitive function. Future studies may further elucidate the consequences and clinical significance of myelin damage in this disease population.

\section{CONCLUSIONS}

Using a myelin-sensitive MR imaging technique, we found that myelin damage may exist in patients with MMD. Although preliminary in many aspects, the results suggest that axonal damage might be more critical for cognitive performance than myelin damage in this disease population.

\section{ACKNOWLEDGMENTS}

We thank Maki Mukawa and Yaeko Furuhashi for performing the gene analysis and the Department of Radiology of Tokyo Medical Clinic for acquiring MR images.

Disclosures: Shoko Hara-RELATED: Grant: Grants-in Aid for Scientific Research "KAKENHI" (grant 19K18406), research grant from the Japanese Society for Neuroradiology. Shigeki Aoki-UNRELATED: Board Membership: Canon, Comments: Canon MR Advisory Board; Grants/Grants Pending: Medi-Physics, Daiichi Sankyo, Eisai, FUJIFILM Toyama Chemical Co, Guerbet, Comments: grant for diagnostic radiology from Medi-Physics, Daiichi Sankyo, Eisai, FUJIFILM Toyama Chemical Co, Guerbet*; Payment for Lectures Including Service on Speakers Bureaus: Bayer Yakuhin, Canon, FUJIFILM Toyama Chemical Co, Eisai, Guerbet, GE Healthcare, Medi-Physics, Siemens, Daiichi Sankyo, Comments: payment for lectures/Chair from Bayer Yakuhin, Canon, FUJIFILM Toyama Chemical Co, Eisai, Guerbet, GE Healthcare, Medi-Physics, Siemens, Daiichi Sankyo; Payment for Manuscript Preparation: MIWA-SHOTEN, Gakken Medical Shujunsha, Comments: payment as Journal Editorial Board member of MIWASHOTEN, Gakken Medical Shujunsha. *Money paid to the institution.

\section{REFERENCES}

1. Farkas E, Luiten PG, Bari F. Permanent, bilateral common carotid artery occlusion in the rat: a model for chronic cerebral hypoperfusionrelated neurodegenerative diseases. Brain Res Rev 2007;54:162-80 CrossRef Medline

2. Kuroda S, Houkin K. Moyamoya disease: current concepts and future perspectives. Lancet Neurol 2008;7:1056-66 CrossRef Medline

3. Hara S, Tanaka Y, Ueda Y, et al. Noninvasive evaluation of CBF and perfusion delay of Moyamoya disease using arterial spin-labeling MRI with multiple postlabeling delays: comparison with ${ }^{15} \mathrm{O}$-gas PET and DSC-MRI. AJNR Am J Neuroradiol 2017;38:696-702 CrossRef Medline

4. Hirai S, Inaji M, Tanaka $\mathrm{Y}$, et al. Correlation between clinical presentations and hemodynamic parameters measured by dynamic susceptibility contrast magnetic resonance imaging in adult patients with Moyamoya disease. J Stroke Cerebrovasc Dis 2017;26:2814-20 CrossRef Medline

5. Kazumata K, Tha KK, Narita H, et al. Chronic ischemia alters brain microstructural integrity and cognitive performance in adult Moyamoya disease. Stroke 2015;46:354-60 CrossRef Medline

6. Hara S, Hori M, Murata S, et al. Microstructural damage in normalappearing brain parenchyma and neurocognitive dysfunction in adult Moyamoya disease. Stroke 2018;49:2504-07 CrossRef Medline

7. Kazumata K, Tha KK, Narita H, et al. Characteristics of diffusional kurtosis in chronic ischemia of adult Moyamoya disease: comparing diffusional kurtosis and diffusion tensor imaging. AJNR Am J Neuroradiol 2016;37:1432-39 CrossRef Medline

8. Stikov N, Campbell JS, Stroh T, et al. In vivo histology of the myelin g-ratio with magnetic resonance imaging. Neuroimage 2015;118: 397-405 CrossRef Medline

9. Helms G, Dathe H, Kallenberg K, et al. High-resolution maps of magnetization transfer with inherent correction for RF inhomogeneity and T1 relaxation obtained from 3D FLASH MRI. Magn Reson Med 2008;60:1396-1407 CrossRef Medline

10. Hori M, Hagiwara A, Fukunaga I, et al. Application of quantitative microstructural MR imaging with atlas-based analysis for the 
spinal cord in cervical spondylotic myelopathy. Sci Rep 2018;8:5213 CrossRef Medline

11. Kamagata K, Zalesky A, Yokoyama K, et al. MR g-ratio-weighted connectome analysis in patients with multiple sclerosis. Sci Rep 2019;9:13522 CrossRef Medline

12. Hagiwara A, Hori M, Yokoyama K, et al. Analysis of white matter damage in patients with multiple sclerosis via a novel in vivo MR method for measuring myelin, axons, and g-ratio. AJNR Am J Neuroradiol 2017;38:1934-40 CrossRef Medline

13. Zhang H, Schneider T, Wheeler-Kingshott CA, et al. NODDI: practical in vivo neurite orientation dispersion and density imaging of the human brain. Neuroimage 2012;61:1000-16 CrossRef Medline

14. Rushton W. A theory of the effects of fibre size in medullated nerve. J Physiol (Lond) 1951;115:101-22 CrossRef Medline

15. Research Committee on the Pathology and Treatment of Spontaneous Occlusion of the Circle of Willis; Health Labour Sciences Research Grant for Research on Measures for Infractable Diseases. Guidelines for diagnosis and treatment of Moyamoya disease (spontaneous occlusion of the circle of Willis). Neurol Med Chir (Tokyo) 2012;52:245-66 CrossRef Medline

16. Morrell GR, Schabel MC. An analysis of the accuracy of magnetic resonance flip angle measurement methods. Phys Med Biol 2010; 55:6157-74 CrossRef Medline

17. Mohammadi S, Carey D, Dick F, et al. Whole-brain in-vivo measurements of the axonal g-ratio in a group of 37 healthy volunteers. Front Neurosci 2015;9:441 CrossRef Medline

18. Campbell JS, Leppert IR, Narayanan S, et al. Promise and pitfalls of g-ratio estimation with MRI. NeuroImage 2018;182:80-96 CrossRef Medline

19. Smith SM, Jenkinson M, Woolrich MW, et al. Advances in functional and structural MR image analysis and implementation as FSL. Neuroimage 2004;23(Suppl 1):S208-19 CrossRef Medline

20. Andersson JL, Skare S, Ashburner J. How to correct susceptibility distortions in spin-echo echo-planar images: application to diffusion tensor imaging. Neuroimage 2003;20:870-88 CrossRef Medline

21. Jenkinson M, Beckmann CF, Behrens TE, et al. FSL. Neuroimage 2012;62:782-90 CrossRef Medline

22. Hagiwara A, Kamagata K, Shimoji K, et al. White matter abnormalities in multiple sclerosis evaluated by quantitative synthetic MRI, diffusion tensor imaging, and neurite orientation dispersion and density imaging. AJNR Am J Neuroradiol 2019;40:1642-48 CrossRef Medline

23. Desikan RS, Segonne F, Fischl B, et al. An automated labeling system for subdividing the human cerebral cortex on MRI scans into gyral based regions of interest. Neuroimage 2006;31:968-80 CrossRef Medline
24. Mutsaerts H, van Dalen JW, Heijtel DF, et al. Cerebral perfusion measurements in elderly with hypertension using arterial spin labeling. PLoS One 2015;10:e0133717 CrossRef Medline

25. Kurumatani T, Kudo T, Ikura Y, et al. White matter changes in the gerbil brain under chronic cerebral hypoperfusion. Stroke 1998;29: 1058-62 CrossRef Medline

26. Lakhani B, Hayward KS, Boyd LA. Hemispheric asymmetry in myelin after stroke is related to motor impairment and function. Neuroimage Clin 2017;14:344-53 CrossRef Medline

27. Hara S, Hori M, Ueda R, et al. Unraveling specific brain microstructural damage in Moyamoya disease using diffusion magnetic resonance imaging and positron emission tomography. J Stroke Cerebrovasc Dis 2019;28:1113-25 CrossRef Medline

28. Kazumata K, Tha KK, Tokairin K, et al. Brain structure, connectivity, and cognitive changes following revascularization surgery in adult Moyamoya disease. Neurosurgery 2019;85:E943-52 CrossRef Medline

29. Roder C, Haas P, Fudali M, et al. Neuropsychological impairment in adults with moyamoya angiopathy: preoperative assessment and correlation to MRI and H215O PET. Neurosurgical Review 2019 Nov 14. [Epub ahead of print] CrossRef Medline

30. Hosoda C, Nariai T, Ishiwata K, et al. Correlation between focal brain metabolism and higher brain function in patients with Moyamoya disease. Int J Stroke 2010;5:367-73 CrossRef Medline

31. Sato Y, Ito K, Ogasawara K, et al. Postoperative increase in cerebral white matter fractional anisotropy on diffusion tensor magnetic resonance imaging is associated with cognitive improvement after uncomplicated carotid endarterectomy: tract-based spatial statistics analysis. Neurosurgery 2013;73:592-98; discussion 598-99 CrossRef Medline

32. Hara $S$, Hori M, Inaji M, et al. Regression of white matter hyperintensity after indirect bypass surgery in a patient with Moyamoya disease. Magn Reson Med Sci 2019;18:247-48 CrossRef Medline

33. Duhamel G, Prevost VH, Cayre M, et al. Validating the sensitivity of inhomogeneous magnetization transfer (ihMT) MRI to myelin with fluorescence microscopy. Neuroimage 2019;199:289-303 CrossRef Medline

34. Maekawa T, Hagiwara A, Hori M, et al. Effect of gadolinium on the estimation of myelin and brain tissue volumes based on quantitative synthetic MRI. AJNR Am J Neuroradiol 2019;40:231-37 CrossRef Medline

35. Saccenti L, Hagiwara A, Andica C, et al. Myelin measurement using quantitative magnetic resonance imaging: a correlation study comparing various imaging techniques in patients with multiple sclerosis. Cells 2020;9:393 CrossRef Medline

36. Hagiwara A, Hori M, Kamagata K, et al. Myelin measurement: comparison between simultaneous tissue relaxometry, magnetization transfer saturation index, and $\mathrm{T} 1 \mathrm{w} / \mathrm{T} 2 \mathrm{w}$ ratio methods. Sci Rep 2018;8:10554 CrossRef 